\begin{tabular}{|c|c|}
\hline Citation/Reference & 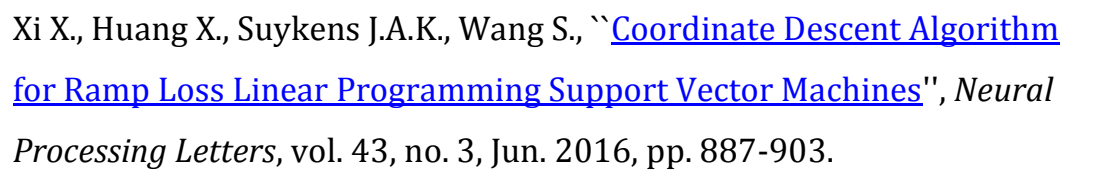 \\
\hline Archived version & $\begin{array}{l}\text { Author manuscript: the content is identical to the content of the } \\
\text { published paper, but without the final typesetting by the publisher }\end{array}$ \\
\hline Published version & $\begin{array}{l}\text { insert link to the published version of your paper } \\
\text { http://dx.doi.org/10.1007/s11063-015-9456-z }\end{array}$ \\
\hline Journal homepage & http://www.springer.com/computer/ai/journal/11063. \\
\hline IR & url in Lirias https://lirias.kuleuven.be/handle/123456789/503160 \\
\hline
\end{tabular}

(article begins on next page) 


\title{
Coordinate Descent Algorithm for Ramp Loss Linear Programming Support Vector Machines
}

\author{
Xiangming Xi • Xiaolin Huang • Johan A.K. \\ Suykens · Shuning Wang
}

Received: date / Accepted: date

\begin{abstract}
In order to control the effects of outliers in training data and get sparse results, Huang et al. [15] proposed the ramp loss linear programming support vector machine (ramp-LPSVM). This combination of $l_{1}$ regularization and ramp loss does not only lead to the sparsity of parameters in decision functions, but also limits the effects of outliers with a maximal penalty. However, due to its non-convexity, the computational cost to achieve a satisfying solution is often expensive. In this paper, we propose a modified coordinate descent algorithm, which deals with a series of one-variable piecewise linear subproblems. Considering that the obtained subproblems are DC programming problems, we linearize the concave part of the objective functions and solve the obtained convex problems. To test the performances of the proposed algorithm, numerical experiments have been carried out and analysed on benchmark data sets. To enhance the sparsity and robustness, the experiments are initialized from C-SVM solutions. The results confirm its excellent performances in classification accuracy, robustness and efficiency in computation.
\end{abstract}

Keywords Support Vector Machines $\cdot$ Ramp Loss $\cdot l_{1}$ Regularization · Coordinate Descent Algorithm

Xiangming Xi ( $ه)$

Department of Automation, Tsinghua National Laboratory for Information Science and Technology (TNList), Tsinghua University, Beijing, 100084, China.

Tel.: +86-10-62785047

Fax: +86-10-62786911

E-mail: xxm10@mails.tsinghua.edu.cn

Xiaolin Huang

Department of Electrical Engineering, ESAT-STADIUS, KU Leuven, Kasteelpark Arenberg 10, B-3001, Leuven, Belgium

E-mail: xiaolin.huang@esat.kuleuven.be

Johan A.K. Suykens

Department of Electrical Engineering, ESAT-STADIUS, KU Leuven, Kasteelpark Arenberg 10, B-3001, Leuven, Belgium

E-mail: johan.suykens@esat.kuleuven.be

Shuning Wang

Department of Automation, Tsinghua National Laboratory for Information Science and Technology (TNList), Tsinghua University, Beijing, 100084, China.

E-mail: swang@mail.tsinghua.edu.cn 


\section{Introduction}

Ever since the proposal of support vector machines (SVM) in [5] and [10], SVM has become one of the most useful tools in classification. For a classification problem, our purpose is to find an optimal decision function to separate the training data, say $\left\{\left(\mathbf{x}_{i}, y_{i}\right)\right\}_{i=1}^{m}$, where $\mathbf{x}_{i} \in \mathbb{R}^{n}$ is a sample point and $y_{i} \in\{-1,+1\}$ is the corresponding class label. A generalized formulation for SVM can be written as follows,

$$
\begin{aligned}
& \min _{\zeta, \mathrm{g}, b} \mu\|\mathrm{g}\|_{\mathrm{K}}^{2}+\frac{1}{m} \sum_{i=1}^{m} \mathrm{~L}\left(\zeta_{i}\right) \\
& \text { s.t. } y_{i}\left(\mathrm{~g}\left(\mathbf{x}_{i}\right)+b\right) \geqslant 1-\zeta_{i}, i=1, \cdots, m, \\
& \quad \zeta \geqslant 0,
\end{aligned}
$$

where $\mathrm{g}$ belongs to the Reproducing Kernel Hilbert Space induced by the Mercer kernel $\mathrm{K}$ with norm $\|\cdot\|_{\mathrm{K}}[2], \zeta \in \mathbb{R}^{m}$ are slack variables, $\mathrm{L}\left(\zeta_{i}\right)$ represents the loss function, $b \in \mathbb{R}$ is the offset, and $\mu$ is the regularization parameter. The objective 11a is of great importance to the performance of the classification ability. The first term, $\|\mathrm{g}\|_{\mathrm{K}}^{2}$, is known as a regularization, of which the common forms include 1-norm ( $l_{1}$ regularization [11]), 2norm $\left(l_{2}\right.$ regularization [10]), or sometimes the $\infty$-norm $\left(l_{\infty}\right.$ regularization [18]). And the commonly used loss functions include the hinge loss $\left(\mathrm{L}_{1}\right.$ loss [36]), squared hinge loss $\left(\mathrm{L}_{2}\right.$ loss [35]) and least square loss [30].

During the development of SVMs, much effort has been made on theoretical analysis of different combinations of regularization forms and loss functions in literature, and most discussions are concerning $l_{2}$ regularized SVMs. Chang et al. [8] propose a fast convergent algorithm based on a sufficient decreasing condition and a modified Newton method. Another technique they use to address the non twice differentiability of the squared hinge loss function is the generalized Hessian matrix proposed by Mangasarian [21]. Other discussions related to $l_{2}$ regularization can be found in [29] and [17] on non-parallel twin support vector machines and multi-class SVMs.

Besides, $l_{1}$ regularization attracts much attention for its sparsity. However, studies concerning $l_{1}$ regularization require the loss function to be differentiable or even twice differentiable, so that the mature gradient based techniques can be applied and convergence analysis could be conducted. Mangasarian [22] investigates the minimization of an $l_{1}$ regularized linear loss problem in the primal form. By obtaining the exact solution to an unconstrained squared hinge function, Fung and Mangasarian [12] propose a coordinate descent algorithm which exploits a generalized Newton method. Schmidt et al. [27] put forward two methods to address the non-differentiability of the $l_{1}$ regularized minimization with continuous and twice differentiable loss function, by smoothing and reformulating the problem as a non-negatively constrained optimization problem. Other discussions include [11, 28, 31,35], etc.

As the hinge loss, squared hinge loss and least square loss all enjoy convexity, they have been investigated by many researchers. However, when extreme outliers exist in training data, the choice of ramp loss will improve the robustness of SVMs against outliers and lead to better performances [4, 25]. The first algorithm for binary SVM with ramp loss is proposed by $\mathrm{Xu}$ et al. [34], who utilize semi-definite programming relaxation. Wu and Liu present the theoretical analysis of the ramp loss SVM and investigate its advantages in sparsity and robustness in [33]. Later researchers reformulate SVM with ramp loss as mixed integer nonlinear programming problems, and then use a branch and bound method [19], 
heuristic algorithms [6] or commercial softwares [7] to obtain a solution. Another method proposed by Collobert et al. in [9] is based on transforming the non-convex ramp loss SVM into a series of convex problems which can be solved by concave-convex procedure. Similar technique is utilized in [32] on the smooth ramp loss SVMs. In addition, Huang et al. [15] propose the ramp loss linear programming support vector machine (ramp-LPSVM). After investigating the formulation of ramp-LPSVM and its theoretical properties, they establish a global search algorithm, which is based on algorithms for DC problems [1], and Hill Detouring Method for concave optimization [16]. Though numerical results confirm that this algorithm outperforms traditional C-SVM in accuracy and robustness, the computational cost for a global search is more expensive. Even though, most of the algorithms mentioned above fail to solve large-scale problems, and could not guarantee the optimality. As interested in optimizing the problem with better performances in efficiency, we will discuss a fast convergent algorithm for ramp-LPSVM in this paper, i.e., a modified coordinate descent algorithm (CDA). Besides the analysis on convergence, its performances on accuracy and robustness are verified by numerical experiments.

The remainder of this paper is organized as follows. Section 2 describes the general concept of coordinate descent algorithm, and then we propose the algorithm for ramp-LPSVM, together with the formulation and convergence analysis of subproblems. The implementation issues are discussed in Section 3 Experiments concerning the comparison of CDA with other algorithms can be found in Section 4 while Section 5 concludes this paper.

\section{Coordinate Descent Algorithm for Ramp-LPSVM}

\subsection{Coordinate Descent Algorithm}

The coordinate descent algorithm is an efficient method for multi-variate optimization. It originates from the research of Hildreth [13], which solves unconstrained quadratic programming problems. Unlike other methods, the main concept of CDA is to optimize one single entry of the independent variables each time, so that the original problem is reduced to a series of one-variable subproblems. If they are convex and differentiable, the subproblems can be efficiently solved with mature techniques. After each entry is optimized iteratively, the algorithm eventually converges under the given assumption of continuity and differentiability [20]. The general framework of CDA is demonstrated in Algorithm 1 .

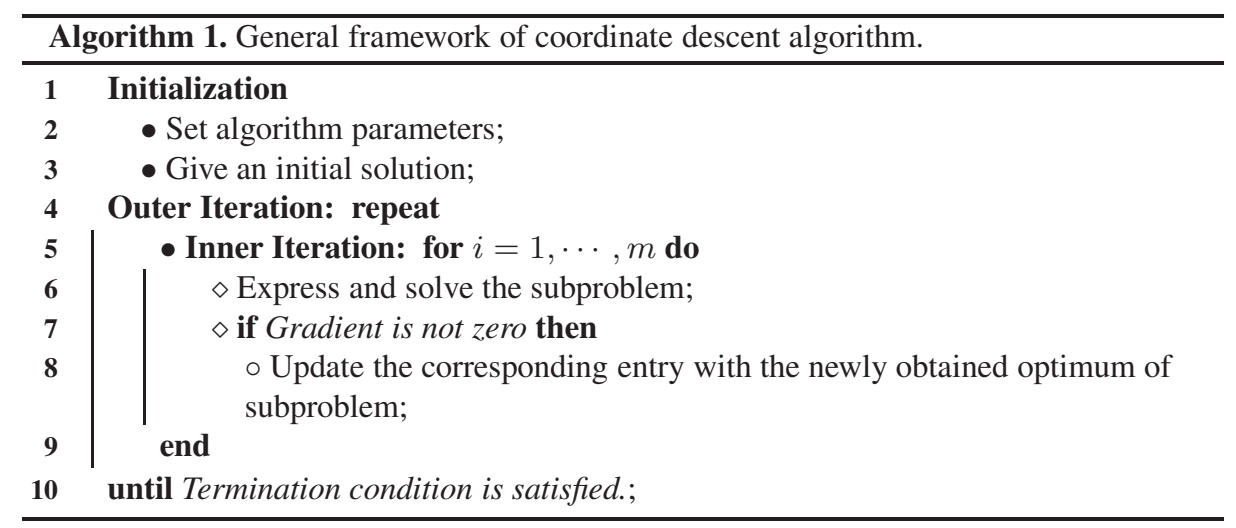


The coordinate descent algorithm is first introduced to support vector machines by Mangasarian and Musicant [23]. As the problem discussed refers to $l_{2}$ regularized problems, the optimization method performs well. Since then, researchers have extended its application to more complex SVM formulations, such as $l_{2}$ regularized SVMs with hinge loss [14], squared hinge loss [8], least square loss [11], and $l_{1}$ regularized problems with logistic loss [35] and so on.

Most of the previous studies concerning coordinate descent algorithm focus on optimizing differentiable SVM problems. Generally speaking, the main computational cost is up to the complexity of the method for solving subproblems, and for a general problem, the cost is often so expensive that it makes CDA applicable to small or medium scaled problems. In order to improve the efficiency of CDA, Chang et al. [8] and Hsieh et al. [14] propose some useful tricks to achieve fast convergence of their algorithms for large-scale SVM problems with linear mapping of the training data. Even though, few attentions are paid on solving $l_{1}$ regularized SVMs with non-differentiable losses in literature. Considering that rampLPSVM has good performances in robustness and sparsity, we are interested in proposing a fast convergent coordinate descent algorithm.

As stated in [15], ramp-LPSVM can be formulated as follows,

$$
\min _{\boldsymbol{\alpha} \geqslant 0, b} \mathrm{f}(\boldsymbol{\alpha}, b)=\mu \sum_{i=1}^{m} \alpha_{i}+\frac{1}{m} \sum_{i=1}^{m} \operatorname{Lramp}\left(1-y_{i}\left(\mathrm{~g}\left(\boldsymbol{\alpha}, \mathbf{x}_{\boldsymbol{i}}\right)+b\right)\right),
$$

where $\operatorname{Lramp}(u)=\max \{u, 0\}-\max \{u-1,0\}$ is the loss function, $\mathrm{g}(\boldsymbol{\alpha}, \mathbf{x})=\sum_{j=1}^{m} \alpha_{j} y_{j}$ $\mathrm{K}\left(\mathbf{x}, \mathbf{x}_{j}\right)$ is the projection function, $\mathrm{K}(\cdot)$ is the kernel function and $b$ is the offset.

The non-convexity and non-concavity of $\operatorname{Lramp}(u)$ make the standard coordinate descent algorithm fail to guarantee the optimality of the final solution. Denote $\xi=\left(\alpha^{T}, b\right)^{T}$, then we provide the compact expression of problem (2) as follows.

$$
\begin{aligned}
& \min _{\xi} f(\xi)=\mathbf{e}_{0}^{T} \xi+\frac{1}{m} \sum_{i=1}^{m} \max \left\{1+\mathbf{c}_{i}^{T} \xi, 0\right\}-\frac{1}{m} \sum_{i=1}^{m} \max \left\{\mathbf{c}_{i}^{T} \xi, 0\right\} \\
& \text { s.t. } \xi_{i} \geqslant 0, i=1, \cdots, m,
\end{aligned}
$$

where $\mathbf{e}_{\mathbf{0}}=(\underbrace{\mu, \cdots, \mu}_{m}, 0)^{T}, \mathbf{C}=\left(\mathbf{c}_{\mathbf{1}}^{T}, \mathbf{c}_{\mathbf{2}}^{T}, \cdots, \mathbf{c}_{\boldsymbol{m}}^{T}\right)$ with

$$
c_{i j}=\left\{\begin{array}{ll}
-y_{i} y_{j} \mathrm{~K}\left(\mathbf{x}_{i}, \mathbf{x}_{j}\right), & j=1, \cdots, m \\
-y_{i}, & j=m+1
\end{array}, i=1, \cdots, m .\right.
$$

Denote the variable to be optimized by $\xi_{k, j} \in \mathbb{R}^{m}$, where the first subscript " $k$ " represents the $k$ th outer iteration in Algorithm 1 , and the second " $j$ " stands for the $j$ th entry. Denote the obtained solution after the $k$ th outer iteration by $\xi_{k+1}$. To minimize (3) over the $j$ th entry of $\xi_{k, j}$, denoted by $\xi_{k, j}^{(j)}$, we express the objective in the following way,

$$
\begin{aligned}
& \mathrm{h}\left(z ; \boldsymbol{\xi}_{k, j}\right) \\
= & \mathrm{f}\left(\boldsymbol{\xi}_{k, j}+\mathbf{e}_{\boldsymbol{j}}\left(z-\boldsymbol{\xi}_{k, j}^{(j)}\right)\right) \\
= & \mathbf{e}_{\mathbf{0}}^{T}\left(\boldsymbol{\xi}_{k, j}+\mathbf{e}_{\boldsymbol{j}}\left(z-\boldsymbol{\xi}_{k, j}^{(j)}\right)\right)+\sum_{i=1}^{m} \max \left\{1+\mathbf{c}_{i}^{T} \boldsymbol{\xi}_{k, j}+\mathbf{c}_{\boldsymbol{i}}^{T} \mathbf{e}_{\boldsymbol{j}}\left(z-\boldsymbol{\xi}_{k, j}^{(j)}\right), 0\right\} / m \\
& -\sum_{i=1}^{m} \max \left\{\mathbf{c}_{i}^{T} \boldsymbol{\xi}_{k, j}+\mathbf{c}_{\boldsymbol{i}}^{T} \mathbf{e}_{\boldsymbol{j}}\left(z-\boldsymbol{\xi}_{k, j}^{(j)}\right), 0\right\} / m \\
= & \mathbf{e}_{\mathbf{0}}^{T} \mathbf{e}_{\boldsymbol{j}} z+\mathbf{e}_{\mathbf{0}}^{T} \boldsymbol{\xi}_{k, j}-\mathbf{e}_{\mathbf{0}}^{T} \mathbf{e}_{\boldsymbol{j}} \boldsymbol{\xi}_{k, j}^{(j)}+\left(\sum_{i=1}^{m} \max \left\{\mathbf{c}_{\boldsymbol{i}}^{T} \mathbf{e}_{\boldsymbol{j}} z+1+\mathbf{c}_{i}^{T} \boldsymbol{\xi}_{k, j}-\mathbf{c}_{\boldsymbol{i}}^{T} \mathbf{e}_{\boldsymbol{j}} \xi_{k, j}^{(j)}, 0\right\}\right. \\
& \left.-\sum_{i=1}^{m} \max \left\{\mathbf{c}_{\boldsymbol{i}}^{T} \mathbf{e}_{\boldsymbol{j}} z+\mathbf{c}_{i}^{T} \boldsymbol{\xi}_{k, j}-\mathbf{c}_{\boldsymbol{i}}^{T} \mathbf{e}_{\boldsymbol{j}} \xi_{k, j}^{(j)}, 0\right\}\right) / m
\end{aligned}
$$


So the corresponding subproblem can be written as,

$$
\min _{z \geqslant 0} \mathrm{~h}\left(z ; \xi_{k, j}\right)
$$

where the nonnegative constraint should be neglected when $j=m+1$ (since $b \in \mathbb{R}$ ).

The outline of the proposed coordinate descent algorithm is presented in Algorithm 2 . Since we are interested in reduction of objective values, the condition in Line 9 of Algorithm 2 ensures the monotonically decrease.

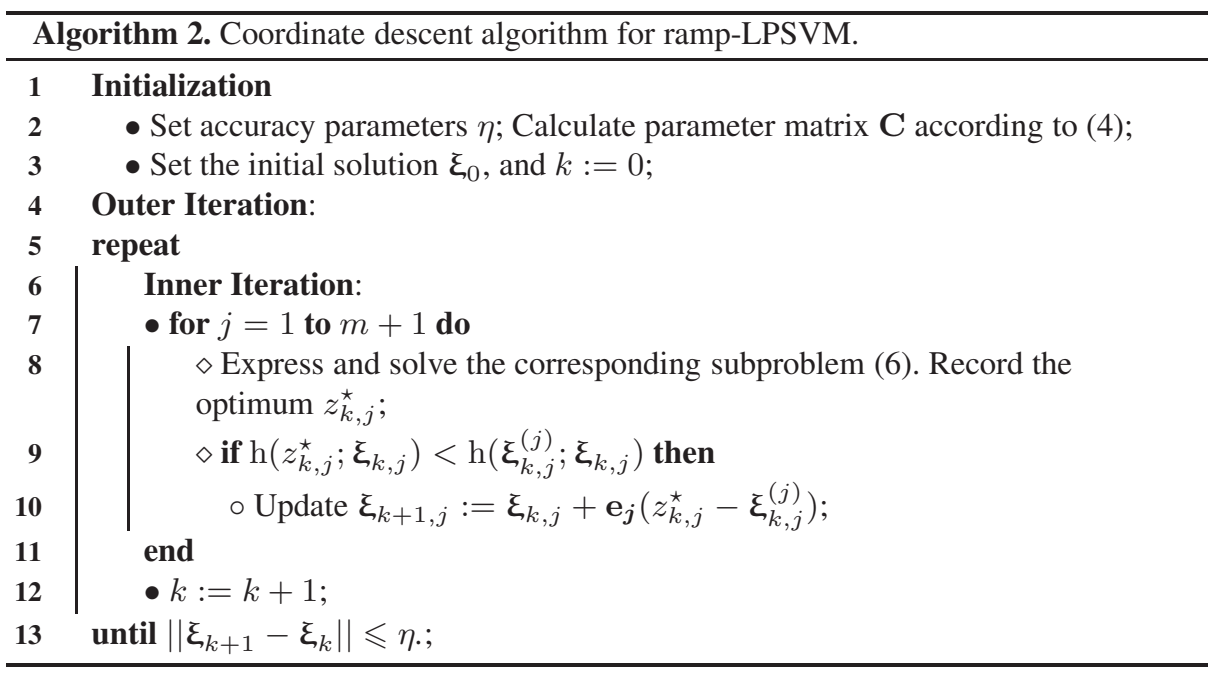

\section{Algorithm Implementation}

The framework of the proposed coordinate descent algorithm is given in the previous section. More detailed implementation issues should be carefully considered so that the algorithm can work to its most. In this section, we will focus on the following issues: the minimization of subproblems, the choice of optimized coordinates and so on.

\subsection{Solving Subproblems}

Recall the subproblem (6). Unlike a general nonlinear optimization problem, the one-variable piecewise linearity makes it convenient to be solved. A simple method to obtain an optimum is to calculate and compare the objective values of all feasible non-differentiable points of $\mathrm{h}(z)$ as well as the bounds. It works well when the problem scale is not large, but as the problem size grows, the computational cost will significantly increase. In order to solve the subproblem more efficiently, we should reformulate the non-convex part of the objective function (5).

Considering that $\mathrm{h}\left(z ; \boldsymbol{\xi}_{k, j}\right)$ is a DC function, we express it as

$$
\mathrm{h}\left(z ; \xi_{k, j}\right)=\overline{\mathrm{h}}\left(z ; \xi_{k, j}\right)+\tilde{\mathrm{h}}\left(z ; \xi_{k, j}\right),
$$


i.e., a summation of a convex function $\overline{\mathrm{h}}\left(z ; \xi_{k, j}\right)$ and a concave function $\tilde{\mathrm{h}}\left(z ; \xi_{k, j}\right)$, where

$$
\begin{aligned}
\overline{\mathrm{h}}\left(z ; \xi_{k, j}\right)= & \mathbf{e}_{\mathbf{0}}^{T} \mathbf{e}_{\boldsymbol{j}} z+\mathbf{e}_{\mathbf{0}}^{T} \boldsymbol{\xi}_{k, j}-\mathbf{e}_{\mathbf{0}}^{T} \mathbf{e}_{\boldsymbol{j}} \boldsymbol{\xi}_{k, j}^{(j)} \\
& +\sum_{i=1}^{m} \max \left\{\mathbf{c}_{\boldsymbol{i}}^{T} \mathbf{e}_{\boldsymbol{j}} z+1+\mathbf{c}_{i}^{T} \boldsymbol{\xi}_{k, j}-\mathbf{c}_{\boldsymbol{i}}^{T} \mathbf{e}_{\boldsymbol{j}} \xi_{k, j}^{(j)}, 0\right\} / m, \\
\tilde{\mathrm{h}}\left(z ; \xi_{k, j}\right)= & -\sum_{i=1}^{m} \max \left\{\mathbf{c}_{\boldsymbol{i}}^{T} \mathbf{e}_{\boldsymbol{j}} z+\mathbf{c}_{i}^{T} \boldsymbol{\xi}_{k, j}-\mathbf{c}_{\boldsymbol{i}}^{T} \mathbf{e}_{\boldsymbol{j}} \xi_{k, j}^{(j)}, 0\right\} / m .
\end{aligned}
$$

The linearization of $\tilde{\mathrm{h}}(\cdot)$ will lead to a convex subproblem, which can be solved efficiently. Thus, we give the partial first-order linearization of $\mathrm{h}\left(z ; \xi_{k, j}\right)$ at $z_{0}$ as follows,

$$
\hat{\mathrm{h}}\left(z ; \xi_{k, j}\right)=\overline{\mathrm{h}}\left(z ; \xi_{k, j}\right)+\tilde{\mathrm{h}}\left(z_{0} ; \xi_{k, j}\right)+\tilde{\mathrm{h}}^{\prime}\left(z_{0} ; \xi_{k, j}\right)\left(z-z_{0}\right),
$$

where $\tilde{\mathrm{h}}^{\prime}\left(z_{0} ; \boldsymbol{\xi}_{k, j}\right)$ is the first order derivative of $\tilde{\mathrm{h}}$ over $z$ at $z_{0}$. It is noticeable that $\tilde{\mathrm{h}}$ is not differentiable at some points, so we will adopt the subgradient method. For any $z \in \mathbb{R}$, the first order derivative of $\tilde{\mathrm{h}}\left(z ; \xi_{k, j}\right)$ is based on

$$
\begin{aligned}
& \tilde{\mathrm{h}}^{\prime}\left(z ; \boldsymbol{\xi}_{k, j}\right)_{+}=-\left(\sum_{i \in S_{1}} \mathbf{c}_{\boldsymbol{i}}^{T} \mathbf{e}_{\boldsymbol{j}}+\sum_{i \in S_{2}} \max \left\{\mathbf{c}_{\boldsymbol{i}}^{T} \mathbf{e}_{\boldsymbol{j}}, 0\right\}\right) / m, \\
& \tilde{\mathrm{h}}^{\prime}\left(z ; \boldsymbol{\xi}_{k, j}\right)_{-}=-\left(\sum_{i \in S_{1}} \mathbf{c}_{\boldsymbol{i}}^{T} \mathbf{e}_{\boldsymbol{j}}+\sum_{i \in S_{2}} \min \left\{\mathbf{c}_{\boldsymbol{i}}^{T} \mathbf{e}_{\boldsymbol{j}}, 0\right\}\right) / m,
\end{aligned}
$$

where $S_{1}=\left\{i \mid \mathbf{c}_{\boldsymbol{i}}^{T} \mathbf{e}_{\boldsymbol{j}} z+\mathbf{c}_{i}^{T} \boldsymbol{\xi}_{k, j}-\mathbf{c}_{\boldsymbol{i}}^{T} \mathbf{e}_{\boldsymbol{j}} \xi_{k, j}^{(j)}>0, i=1, \cdots, m\right\}, S_{2}=\left\{i \mid \mathbf{c}_{\boldsymbol{i}}^{T} \mathbf{e}_{\boldsymbol{j}} z+\right.$ $\left.\mathbf{c}_{i}^{T} \xi_{k, j}-\mathbf{c}_{\boldsymbol{i}}^{T} \mathbf{e}_{j} \xi_{k, j}^{(j)}=0, i=1, \cdots, m\right\}$. The subscript "+" and "-_" indicate the right and left derivative, respectively. For the non-differentiable points, the subgradient is defined as $\tilde{\mathrm{h}}^{\prime}\left(z ; \xi_{k, j}\right)=\lambda \tilde{\mathrm{h}}^{\prime}\left(z ; \xi_{k, j}\right)_{+}+(1-\lambda) \tilde{\mathrm{h}}^{\prime}\left(z ; \xi_{k, j}\right)_{-}$, where $\lambda \in[0,1]$.

In this way, the subproblem is transformed to a convex problem in $\mathbb{R}$ and the computational cost for an optimum is much less than that when solving problem (6). A useful property of the subgradient of $\hat{\mathrm{h}}$ is provided as follows, which will be exploited in the convergence analysis in the successive section.

Proposition 1 For a convex function $\hat{\mathrm{h}}$ in the form of (7), the subgradient of $\hat{\mathrm{h}}$ at any point $z \in \mathbb{R}$ satisfies

$$
\left\|\hat{\mathrm{h}}^{\prime}\left(z ; \xi_{k, j}\right)\right\| \leqslant 4\|C\|_{1} / m+1 .
$$

Moreover,

$$
\left\|\left.\hat{\mathrm{h}}^{\prime}\left(z ; \boldsymbol{\xi}_{k, j}\right)\right|_{z=z_{0}}\right\| \leqslant\|C\|_{1} / m+1 \text {. }
$$

Proof From the expressions of (7) and (8), we can get the subgradient of $\hat{h}$ at any point $z \in \mathbb{R}$ as follows,

$$
\hat{\mathrm{h}}^{\prime}\left(z ; \boldsymbol{\xi}_{k, j}\right)=\overline{\mathrm{h}}^{\prime}\left(z ; \boldsymbol{\xi}_{k, j}\right)+\tilde{\mathrm{h}}^{\prime}\left(z_{0} ; \boldsymbol{\xi}_{k, j}\right) .
$$

Besides the expression of $\tilde{\mathrm{h}}^{\prime}$ in $[8]$, the $\overline{\mathrm{h}}^{\prime}\left(z ; \xi_{k, j}\right)$ is given as follows,

$$
\begin{aligned}
& \overline{\mathrm{h}}^{\prime}\left(z ; \xi_{k, j}\right)_{+}=\mathbf{e}_{\mathbf{0}}^{T} \mathbf{e}_{\boldsymbol{j}}+\left(\sum_{i \in S_{3}} \mathbf{c}_{\boldsymbol{i}}^{T} \mathbf{e}_{\boldsymbol{j}}+\sum_{i \in S_{4}} \max \left\{\mathbf{c}_{\boldsymbol{i}}^{T} \mathbf{e}_{\boldsymbol{j}}, 0\right\}\right) / m, \\
& \overline{\mathrm{h}}^{\prime}\left(z ; \boldsymbol{\xi}_{k, j}\right)_{-}=\mathbf{e}_{\mathbf{0}}^{T} \mathbf{e}_{\boldsymbol{j}}+\left(\sum_{i \in S_{3}} \mathbf{c}_{\boldsymbol{i}}^{T} \mathbf{e}_{\boldsymbol{j}}+\sum_{i \in S_{4}} \min \left\{\mathbf{c}_{\boldsymbol{i}}^{T} \mathbf{e}_{\boldsymbol{j}}, 0\right\}\right) / m,
\end{aligned}
$$

where $S_{3}=\left\{i \mid \mathbf{c}_{\boldsymbol{i}}^{T} \mathbf{e}_{\boldsymbol{j}} z+1+\mathbf{c}_{i}^{T} \xi_{k, j}-\mathbf{c}_{\boldsymbol{i}}^{T} \mathbf{e}_{\boldsymbol{j}} \xi_{k, j}^{(j)}>0, i=1, \cdots, m\right\}, S_{4}=\{i \mid$ $\left.\mathbf{c}_{\boldsymbol{i}}^{T} \mathbf{e}_{\boldsymbol{j}} z+1+\mathbf{c}_{i}^{T} \xi_{k, j}-\mathbf{c}_{\boldsymbol{i}}^{T} \mathbf{e}_{\boldsymbol{j}} \xi_{k, j}^{(j)}=0, i=1, \cdots, m\right\}$. Then we have

$$
\begin{aligned}
& \hat{\mathrm{h}}^{\prime}\left(z ; \xi_{k, j}\right) \\
= & \mathbf{e}_{\mathbf{0}}^{T} \mathbf{e}_{\boldsymbol{j}}+\sum_{i \in S_{3}} \mathbf{c}_{\boldsymbol{i}}^{T} \mathbf{e}_{\boldsymbol{j}} / m-\sum_{i \in S_{1}} \mathbf{c}_{\boldsymbol{i}}^{T} \mathbf{e}_{\boldsymbol{j}} / m \\
& \left.+\left(\lambda_{1} \sum_{i \in S_{4}} \max \left\{\mathbf{c}_{\boldsymbol{i}}^{T} \mathbf{e}_{\boldsymbol{j}}, 0\right\}+\left(1-\lambda_{1}\right) \sum_{i \in S_{4}} \min \left\{\mathbf{c}_{\boldsymbol{i}}^{T} \mathbf{e}_{\boldsymbol{j}}, 0\right\}\right) / m\right) \\
& -\left(\lambda_{2} \sum_{i \in S_{2}} \min \left\{\mathbf{c}_{\boldsymbol{i}}^{T} \mathbf{e}_{\boldsymbol{j}}, 0\right\}+\left(1-\lambda_{2}\right) \sum_{i \in S_{2}} \max \left\{\mathbf{c}_{\boldsymbol{i}}^{T} \mathbf{e}_{\boldsymbol{j}}, 0\right\} / m\right) .
\end{aligned}
$$


Now we turn to the relation between $S_{i}, i=1, \cdots, 4$. First, the definition of $S_{i}$ leads to that $S_{1} \cap S_{2}=\emptyset$ and $S_{3} \cap S_{4}=\emptyset$. Second, since $S_{1}, S_{2}$ are determined by $z_{0}$, and $S_{3}, S_{4}$ by $z$, if taking $z=z_{0}$, then $S_{1} \subset S_{3}$. By using these two facts, there holds that $\left(S_{3}-S_{1}\right) \cap S_{2} \cap S_{4}=\emptyset$, and that

$$
\begin{aligned}
& \left\|\left.\hat{h}^{\prime}\left(z ; \boldsymbol{\xi}_{k, j}\right)\right|_{z=z_{0}}\right\| \\
= & \left\|\mathbf{e}_{\mathbf{0}}^{T} \mathbf{e}_{\boldsymbol{j}}\right\|+\left\|\sum_{i \in S_{3}-S_{1}} \mathbf{c}_{\boldsymbol{i}}^{T} \mathbf{e}_{\boldsymbol{j}} / m\right\| \\
& \left.+\|\left(\lambda_{1} \sum_{i \in S_{4}} \max \left\{\mathbf{c}_{\boldsymbol{i}}^{T} \mathbf{e}_{\boldsymbol{j}}, 0\right\} / m+\left(1-\lambda_{1}\right) \sum_{i \in S_{4}} \min \left\{\mathbf{c}_{\boldsymbol{i}}^{T} \mathbf{e}_{\boldsymbol{j}}, 0\right\}\right) / m\right) \| \\
& +\left\|\left(\lambda_{2} \sum_{i \in S_{2}} \min \left\{\mathbf{c}_{\boldsymbol{i}}^{T} \mathbf{e}_{\boldsymbol{j}}, 0\right\} / m+\left(1-\lambda_{2}\right) \sum_{i \in S_{2}} \max \left\{\mathbf{c}_{\boldsymbol{i}}^{T} \mathbf{e}_{\boldsymbol{j}}, 0\right\} / m\right)\right\| \\
\leqslant & 1+\sum_{i \in\left(S_{3}-S_{1}\right) \cup S_{2} \cup S_{4}}\left|\mathbf{c}_{\boldsymbol{i}}^{T} \mathbf{e}_{\boldsymbol{j}}\right| / m \\
\leqslant & 1+\left\|C_{1}\right\| / m .
\end{aligned}
$$

If $z \neq z_{0}$, then based on the similar analysis above, we can see that

$$
\begin{aligned}
& \left\|\hat{\mathrm{h}}^{\prime}\left(z ; \boldsymbol{\xi}_{k, j}\right)\right\| \\
\leqslant & 1+\left\|\sum_{i \in S_{3}} \mathbf{c}_{\boldsymbol{i}}^{T} \mathbf{e}_{\boldsymbol{j}} / m\right\|+\left\|\sum_{i \in S_{1}} \mathbf{c}_{\boldsymbol{i}}^{T} \mathbf{e}_{\boldsymbol{j}} / m\right\| \\
& \left.+\|\left(\lambda_{1} \sum_{i \in S_{4}} \max \left\{\mathbf{c}_{\boldsymbol{i}}^{T} \mathbf{e}_{\boldsymbol{j}}, 0\right\} / m+\left(1-\lambda_{1}\right) \sum_{i \in S_{4}} \min \left\{\mathbf{c}_{\boldsymbol{i}}^{T} \mathbf{e}_{\boldsymbol{j}}, 0\right\}\right) / m\right) \| \\
& +\left\|\left(\lambda_{2} \sum_{i \in S_{2}} \min \left\{\mathbf{c}_{\boldsymbol{i}}^{T} \mathbf{e}_{\boldsymbol{j}}, 0\right\} / m+\left(1-\lambda_{2}\right) \sum_{i \in S_{2}} \max \left\{\mathbf{c}_{\boldsymbol{i}}^{T} \mathbf{e}_{\boldsymbol{j}}, 0\right\} / m\right)\right\| \\
\leqslant & 1+4\left\|C_{1}\right\| / m .
\end{aligned}
$$

The last step above is because that though $S_{1}, S_{3}$ may share common elements, all $S_{i}$ are subsets of $\{1, \cdots, m\}$, which leads to the last step by proper relaxation.

As interested in finding the solution which satisfies $\hat{\mathrm{h}}^{\prime}\left(z ; \xi_{k, j}\right)=0$, we will implement the detailed method with gradient (or subgradient) information of the linearized problem, which is illustrated in Algorithm 3

Algorithm 3 exploits the fact that due to the convexity of $\hat{h}$, the possible cases of derivatives at lower and upper bounds are (non-negative, non-negative), (non-positive, nonpositive), (non-positive, non-negative). In the first two cases, the optimum is the lower bound and upper bound, respectively, while only in the third case, an iterative searching procedure should be conducted to locate the optimum. Another issue we should notice is that for rampLPSVM, the value of the second term of $\mathrm{f}$ in problem (2) is within the interval $[0,1]$, so $\xi$ should be bounded by a considerably small positive value. As a result, it is reasonable to set a fixed upper bound, say $b_{u}$, during the searching procedure, while to keep the optimum unchanged.

\subsection{Convergence Analysis}

Theorem 1 Let $\left\{\xi_{k}\right\},\left\{f\left(\xi_{k}\right)\right\}$ be sequences generated by Algorithm 2 then we have the following convergence results.

(1) $\left\{\mathrm{f}\left(\xi_{k}\right)\right\}$ is nonincreasing and it satisfies

$$
\mathrm{f}\left(\boldsymbol{\xi}_{k+1}\right)-\mathrm{f}\left(\boldsymbol{\xi}_{k}\right) \leqslant-\sum_{j \in \hat{S}_{k}} \gamma \beta^{s_{k, j}-1}
$$

where $\hat{S}_{k}=\left\{j \mid z_{k, j}^{\star} \neq \xi_{k, j}^{(j)}\right\}$; 


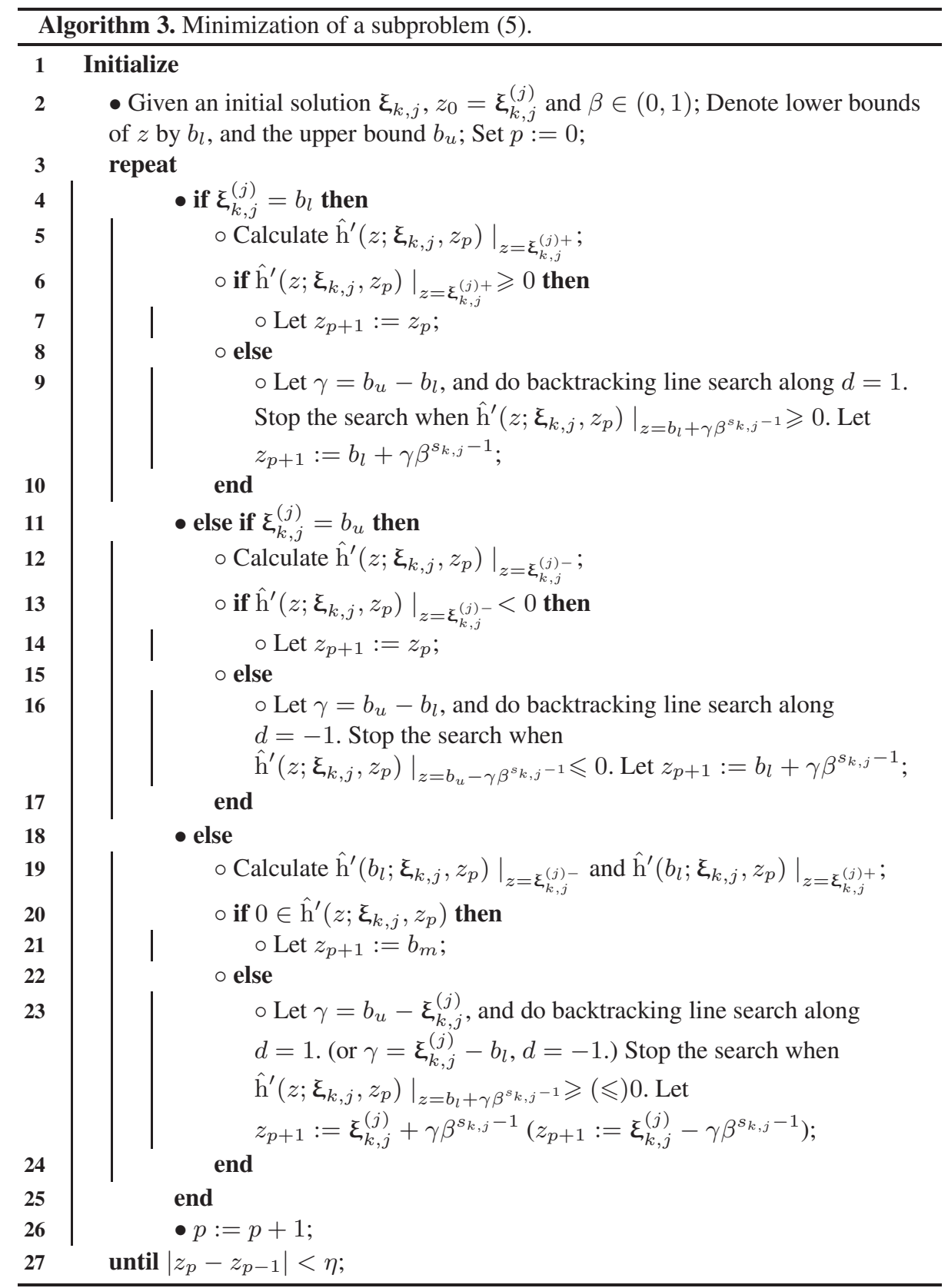


(2) If $\left\{\xi_{k}\right\}_{\mathcal{K}}$ is a convergent subsequence of $\left\{\xi_{k}\right\}$, then $\hat{S}_{k} \rightarrow \emptyset$, and $\left\{\mathrm{f}\left(\xi_{k+1}\right)-\mathrm{f}\left(\xi_{k}\right)\right\} \rightarrow$ 0 ;

Proof (1) Take the $j$-th inner iteration of the $k$-th outer iteration into consideration, and the problem is

$$
\begin{aligned}
& \min _{z} \hat{\mathrm{h}}\left(z ; \xi_{k, j}\right) \\
& \text { s.t. } z \in\left[b_{l}, b_{u}\right] .
\end{aligned}
$$

And due to the concavity of $\tilde{\mathrm{h}}$, there is

$$
\begin{aligned}
& \mathrm{h}\left(z_{k, j}^{\star} ; \xi_{k, j}\right)-\mathrm{h}\left(z_{0} ; \xi_{k, j}\right) \\
= & \overline{\mathrm{h}}\left(z_{k, j}^{\star} ; \xi_{k, j}\right)+\tilde{\mathrm{h}}\left(z_{k, j}^{\star} ; \xi_{k, j}\right)-\left(\overline{\mathrm{h}}\left(z_{0} ; \xi_{k, j}\right)+\tilde{\mathrm{h}}\left(z_{0} ; \boldsymbol{\xi}_{k, j}\right)\right) \\
\leqslant & \hat{\mathrm{h}}\left(z_{k, j}^{\star} ; \boldsymbol{\xi}_{k, j}\right)-\hat{\mathrm{h}}\left(z_{0} ; \xi_{k, j}\right) .
\end{aligned}
$$

Noticed that if $z_{0}=\xi_{k, j}^{(j)}$, then we have $\mathrm{h}\left(z_{k, j-1}^{\star} ; \xi_{k, j-1}\right)=\mathrm{h}\left(z_{0} ; \xi_{k, j}\right)$. This leads to

$$
\mathrm{h}\left(z_{k, j}^{\star} ; \xi_{k, j}\right)-\mathrm{h}\left(z_{k, j-1}^{\star} ; \xi_{k, j-1}\right) \leqslant \hat{\mathrm{h}}\left(z_{k, j}^{\star} ; \xi_{k, j}\right)-\hat{\mathrm{h}}\left(z_{k, j-1}^{\star} ; \xi_{k, j-1}\right) .
$$

Sum up the above inequalities over $j=1, \cdots, n$, then we have

$$
\mathrm{h}\left(z_{k, n}^{\star} ; \boldsymbol{\xi}_{k, n}\right)-\mathrm{h}\left(z_{0} ; \xi_{k, 0}\right) \leqslant \sum_{j=1}^{n}\left(\hat{\mathrm{h}}\left(z_{k, j}^{\star} ; \boldsymbol{\xi}_{k, j}\right)-\hat{\mathrm{h}}\left(\xi_{k, j}^{(j)} ; \boldsymbol{\xi}_{k, j}\right)\right)
$$

which is

$$
\mathrm{f}\left(\boldsymbol{\xi}_{k+1}\right)-\mathrm{f}\left(\boldsymbol{\xi}_{k}\right) \leqslant \sum_{j=1}^{n}\left(\hat{\mathrm{h}}\left(z_{k, j}^{\star} ; \boldsymbol{\xi}_{k, j}\right)-\hat{\mathrm{h}}\left(\boldsymbol{\xi}_{k, j}^{(j)} ; \boldsymbol{\xi}_{k, j}\right)\right) .
$$

Denote $\hat{S}_{k}=\left\{j \mid z_{k, j}^{\star} \neq \xi_{k, j}^{(j)}\right\}$, and $\hat{S}_{k}^{c}$ is its complementary set. Since for any $j \in \hat{S}_{k}^{c}$, it holds that $z_{k, j}^{\star}=\xi_{k, j}^{(j)}$ and $\left.\hat{h}^{\prime}\left(z ; \xi_{k, j}\right)\right|_{z=\xi_{k, j}^{(j)}}\left(z_{k, j}^{\star}-\xi_{k, j}^{(j)}\right)=0$. Then (13) can be expressed as

$$
\begin{aligned}
\mathrm{f}\left(\xi_{k+1}\right)-\mathrm{f}\left(\boldsymbol{\xi}_{k}\right) & \leqslant \sum_{j=1}^{n}\left(\hat{\mathrm{h}}\left(z_{k, j}^{\star} ; \xi_{k, j}\right)-\hat{\mathrm{h}}\left(0 ; \xi_{k, j}\right)\right) \\
& \leqslant\left.\sum_{j \in \hat{S}_{k}} \hat{\mathrm{h}}^{\prime}\left(z ; \xi_{k, j}\right)\right|_{z=\xi_{k, j}^{(j)}}\left(z_{k, j}^{\star}-\xi_{k, j}^{(j)}\right) .
\end{aligned}
$$

However, as $\hat{\mathrm{h}}$ is not differentiable at some points, we need appeal to the subgradient of $\hat{\mathrm{h}}$. Remind the expression of $\hat{\mathrm{h}}$ in (7), and its subgradient from above and from below is defined as

$$
\begin{aligned}
& \left.\hat{\mathrm{h}}^{\prime}\left(z ; \xi_{k, j}\right)\right|_{z \rightarrow \xi_{k, j}^{(j)-}}=\left.\overline{\mathrm{h}}^{\prime}\left(z ; \xi_{k, j}\right)\right|_{z \rightarrow \xi_{k, j}^{(j)-}}-\left.\tilde{\mathrm{h}}^{\prime}\left(z ; \xi_{k, j}\right)\right|_{z=\xi_{k, j}^{(j)}}, \\
& \left.\hat{\mathrm{h}}^{\prime}\left(z ; \xi_{k, j}\right)\right|_{z \rightarrow \xi_{k, j}^{(j)+}}=\left.\overline{\mathrm{h}}^{\prime}\left(z ; \xi_{k, j}\right)\right|_{z \rightarrow \xi_{k, j}^{(j)+}}-\left.\tilde{\mathrm{h}}^{\prime}\left(z ; \xi_{k, j}\right)\right|_{z=\xi_{k, j}^{(j)}},
\end{aligned}
$$

where $\left.\tilde{h}^{\prime}\left(z ; \xi_{k, j}\right)\right|_{z=\xi_{k, j}^{(j)}} ^{(j)}$ is the subgradient of $\tilde{\mathrm{h}}\left(z ; \xi_{k, j}\right)$ at $\xi_{k, j}$.

According to Algorithm 3 if $z_{k, j}^{\star} \neq \xi_{k, j}$, one of the following conditions holds,

- $\xi_{k, j}^{(j)} \in\left(b_{l}, b_{u}\right),\left.\hat{\mathrm{h}}_{-}^{\prime}\left(z ; \xi_{k, j}\right)\right|_{z \rightarrow \xi_{k, j}^{(j)-}}>0$, and $\left.\hat{\mathrm{h}}^{\prime}\left(z ; \xi_{k, j}\right)\right|_{z \rightarrow \xi_{k, j}^{(j)+}}>0$;

- $\xi_{k, j}^{(j)} \in\left(b_{l}, b_{u}\right),\left.\hat{\mathrm{h}}_{-}^{\prime}\left(z ; \xi_{k, j}\right)\right|_{z \rightarrow \xi_{k, j}^{(j)-}}<0$, and $\left.\hat{\mathrm{h}}^{\prime}\left(z ; \xi_{k, j}\right)\right|_{z \rightarrow \xi_{k, j}^{(j)+}}<0 ;$

- $\xi_{k, j}^{(j)}=b_{l},\left.\hat{h}^{\prime}\left(z ; \xi_{k, j}\right)\right|_{z \rightarrow \xi_{k, j}^{(j)+}}<0 ;$ 
$-\xi_{k, j}^{(j)}=b_{u},\left.\hat{h}^{\prime}\left(z ; \xi_{k, j}\right)\right|_{z \rightarrow \xi_{k, j}^{(j)-}}>0$.

In the first and fourth cases, as $\hat{\mathrm{h}}$ is convex, and the subgradient is a convex combination of the limitation from above and below, the optimum to problem $10, z_{k, j}^{\star}$, must be smaller than $\xi_{k, j}^{(j)}$. While in the other cases, $z_{k, j}^{\star}>\xi_{k, j}^{(j)}$.

Define $\Delta_{k} \triangleq-\left.\sum_{j \in \hat{S}_{k}} \hat{\mathrm{h}}^{\prime}\left(z ; \xi_{k, j}\right)\right|_{z=\xi_{k, j}^{(j)}}\left(z_{k, j}^{\star}-\xi_{k, j}^{(j)}\right)$. Using the Armijo rules to search for $z_{k, j}^{\star}$ will lead to

$$
\Delta_{k}=-\sum_{j \in \hat{S}_{k}} \gamma \beta^{s_{k, j}-1}
$$

where $d_{k, j}=\operatorname{sign}\left(\hat{\mathrm{h}}^{\prime}\left(\xi_{k, j}^{(j)} ; \xi_{k, j}\right)\right)$. Then

$$
\mathrm{f}\left(\xi_{k+1}\right)-\mathrm{f}\left(\xi_{k}\right) \leqslant-\sum_{j \in \hat{S}_{k}} \gamma \beta^{s_{k, j}-1} \leqslant 0 .
$$

(2) Let $\left\{\xi_{k}\right\}_{\mathcal{K}}$ be a subsequence of $\left\{\xi_{k}\right\}$ which converges to $\bar{\xi}$. Then since $\left\{f\left(\xi_{k}\right)\right\}$ is non-increasing and is lower bounded, it holds that $\mathrm{f}(\bar{\xi}) \leqslant \liminf _{k \rightarrow \infty, k \in \mathcal{K}} \mathrm{f}\left(\boldsymbol{\xi}_{k}\right)$. Therefore, it implies that $\{\mathrm{f}(\xi)\}_{\mathcal{K}} \rightarrow \mathrm{f}(\bar{\xi})$, and thus, $\left\{\mathrm{f}\left(\xi_{k+1}\right)-\mathrm{f}\left(\xi_{k}\right)\right\} \rightarrow 0$. And then we have

$$
\left\{\Delta_{k}\right\} \rightarrow 0 \text {. }
$$

Suppose that $\left\{\hat{S}_{k}\right\}_{\mathcal{K}}$ is not convergent to $\emptyset$. By a proper selection of $\mathcal{K}$, there exists $j_{k} \in \hat{S}_{k}$, for any $k \geqslant \bar{k}$, where $\bar{k}$ is a positive integer. Then after one outer iteration, the minimization of $\hat{\mathrm{h}}\left(z ; \boldsymbol{\xi}_{k, j}\right)$ is solved with one of the following conditions,

(a) $\xi_{k, j_{k}}^{\left(j_{k}\right)} \in\left(b_{l}, b_{u}\right),\left.0 \notin \hat{\mathrm{h}}^{\prime}\left(z ; \xi_{k, j_{k}}\right)\right|_{z=\xi_{k, j_{k}}^{\left(j_{k}\right)}}$;

(b) $\xi_{k, j_{k}}^{\left(j_{k}\right)}=b_{l},\left.\hat{\mathrm{h}}^{\prime}\left(z ; \xi_{k, j_{k}}\right)\right|_{z=b_{l}^{+}}<0$;

(c) $\xi_{k, j_{k}}^{\left(j_{k}\right)}=b_{u},\left.\hat{\mathrm{h}}^{\prime}\left(z ; \xi_{k, j_{k}}\right)\right|_{z=b_{u}^{-}}>0$.

Moreover, there exists $\epsilon>0$, such that $\Delta_{k}=\left.\sum_{j \in \hat{S}_{k}} \hat{\mathrm{h}}^{\prime}\left(z ; \xi_{k, j}\right)\right|_{z=\xi_{k, j}^{(j)}}\left(z_{k, j}^{\star}-\right.$ $\left.\xi_{k, j}^{(j)}\right) \leqslant\left.\hat{\mathrm{h}}^{\prime}\left(z ; \xi_{k, j}\right)\right|_{z=\xi_{k, j_{k}}^{\left(j_{k}\right)}}\left(z_{k, j_{k}}^{\star}-\xi_{k, j_{k}}^{\left(j_{k}\right)}\right) \leqslant-\epsilon<0$.

By summing up Equation (14) for $k \in \mathcal{K}$, we have

$$
\begin{aligned}
\mathrm{f}\left(\xi_{k}\right)-\mathrm{f}\left(\xi_{1}\right) & \leqslant\left.\sum_{j \in \hat{S}_{k}} \hat{\mathrm{h}}^{\prime}\left(z ; \xi_{k, j}\right)\right|_{z=\xi_{k, j}^{(j)}}\left(z_{k, j}^{\star}-\xi_{k, j}^{(j)}\right) \\
& \leqslant\left.\hat{\mathrm{h}}^{\prime}\left(z ; \xi_{k, j}\right)\right|_{z=\xi_{k, j_{k}}^{\left(j_{k}\right)}}\left(z_{k, j_{k}}^{\star}-\xi_{k, j_{k}}^{\left(j_{k}\right)}\right) \\
& <k \epsilon
\end{aligned}
$$

which implies

$$
\inf _{k \rightarrow \infty, k \in \mathcal{K}} \mathrm{f}\left(\xi_{k}\right)-\mathrm{f}\left(\xi_{1}\right)=-\infty .
$$

A clear contradiction against that $\mathrm{f}(\xi)$ is lower bounded can be seen. Thus, $\left\{\hat{S}_{k}\right\} \rightarrow \emptyset$, and $\left\{\Delta_{k}\right\} \rightarrow 0$.

Theorem 2 Let $\left\{\xi_{k}\right\},\left\{f\left(\xi_{k}\right)\right\}$ be sequences generated by Algorithm 2 If the coefficient matrix $C$ is proper, i.e., $\|C\|_{1}<+\infty$, then $\left\{\mathrm{f}\left(\xi_{k}\right)\right\}$ converges $Q$-linearly over the outer iterations. 
Proof Suppose that there exists a subsequence of $\left\{\boldsymbol{\xi}_{k}\right\}$, denoted by $\left\{\boldsymbol{\xi}_{k}\right\}_{\mathcal{K}}$, which converges to a point $\bar{\xi}$. The condition $\|C\|_{1}<+\infty$ implies that the subgradient of $f$ at any point is proper, and for the problem we discuss, it holds according to Proposition 1 Thus, we have

$$
\mathrm{f}\left(\xi_{k}\right)-\mathrm{f}(\bar{\xi})=\nabla \mathrm{f}(\tilde{\xi})^{T}\left(\xi_{k}-\bar{\xi}\right) \leqslant L\left\|\xi_{k}-\bar{\xi}\right\|,
$$

where $\|\nabla \mathrm{f}\|<L$ and $0<L<\infty$. Moreover, there holds

$$
\begin{aligned}
\left\|\xi_{k}-\bar{\xi}\right\| & \leqslant\left\|\xi_{k+1}-\xi_{k}\right\|+\left\|\xi_{k+1}-\bar{\xi}\right\| \\
& \ldots \lim _{N \rightarrow \infty} \sum_{l=1}^{N}\left\|\xi_{k+l+1}-\xi_{k+l}\right\|+\left\|\xi_{k+N+1}-\bar{\xi}\right\| \\
& \leqslant N_{1} \sup _{l}\left\|\xi_{k+l+1}-\xi_{k+l}\right\| \\
& =N_{1} \sup _{l}\left(\sum_{j \in \hat{S}_{k+l}}\left(\gamma \beta^{s_{k, j}-1} d_{k, j}\right)^{2}\right)^{1 / 2} . \\
& =N_{1} \sup _{l}\left(\sum_{j \in \hat{S}_{k+l}}\left(\gamma \beta^{s_{k, j}-1}\right)^{2}\right)^{1 / 2} / \min _{j \in \hat{S}_{k+l}}\left|\hat{\mathrm{h}}^{\prime}\left(\xi_{k, j}^{(j)} ; \xi_{k, j}\right)\right| \\
& \leqslant N_{2}\left(-\Delta_{k}\right),
\end{aligned}
$$

where $\xi_{k+l} \in\left\{\xi_{k}\right\}_{\mathcal{K}}$, and $0<N_{2}<+\infty$. The third step of the above relation holds since for any points in $\left\{\xi_{k}\right\}_{\mathcal{K}}$, there exists $N_{1}>0$, such that for any $k>N_{1}$ and $\nu>0$, $\left\|\xi_{k}-\bar{\xi}\right\|<\nu$. The last step is conducted following the fact that for any $j \in \hat{S}_{k}$, the subgradient of $\hat{\mathrm{h}}$ is nonzero, and thus, $\inf _{k} \min _{j \in \hat{S}_{k+l}}\left|\hat{\mathrm{h}}^{\prime}\left(\xi_{k, j}^{(j)} ; \xi_{k, j}\right)\right|>0$.

Combining the above two equations leads to

$$
\begin{aligned}
\mathrm{f}\left(\xi_{k}\right)-\mathrm{f}(\bar{\xi}) & \leqslant L\left\|\xi_{k}-\bar{\xi}\right\| \\
& \leqslant L N_{2}\left(-\Delta_{k}\right) \\
& \leqslant L N_{2}\left(\mathrm{f}\left(\xi_{k+1}\right)-\mathrm{f}\left(\xi_{k}\right)\right) .
\end{aligned}
$$

From (22), we can see that

$$
\mathrm{f}\left(\boldsymbol{\xi}_{k+1}\right)-\mathrm{f}(\bar{\xi}) \leqslant \frac{L N_{2}-1}{L N_{2}}\left(\mathrm{f}\left(\xi_{k}\right)-\mathrm{f}(\bar{\xi})\right),
$$

which means $\left\{\mathrm{f}\left(\xi_{k}\right)\right\}$ converges to $\mathrm{f}(\overline{\boldsymbol{\xi}})$ at least Q-linearly over outer iterations.

Though f converges at least Q-linearly over outer iterations, Algorithm 2 might minimize every variables in each outer iteration, and this would take more computational time than that of the theoretical analysis. This is also verified by the numerical experiments in the following section.

\subsection{Other Discussions}

In literature, researchers have already discussed the influence of the updating order of coordinates on experimental performances of CDA. Shalev-Shwartz and Tewari [28] propose a statistic coordinate descent algorithm for $l_{1}$ regularized SVMs, and establish the corresponding convergence analysis. Later, an extension of the coordinate gradient descent algorithm is proposed in [31] to deal with SVM problems of which the objectives consist of smooth functions and separable convex functions. The $\mathrm{l}_{2}$ regularized SVMs with several non-convex losses are discussed in [24]. When it comes to Algorithm 2] we will adopt the statistic order of the updated coordinates, while specifically, we choose the uniform distribution.

The proposed algorithm is sensitive to initial solutions due to the lack of convexity of $\mathrm{f}$. Since $l_{1}$ regularization implies sparsity of SVMs, most of the entries of $\alpha$ will be 0 . A good 
trial is to set the initial solution of $\alpha$ to be 0 . The numerical experiment about the choice of initial solution can be found in Section 4.1. In the experiments, the randomness caused by the choice of coordinate orders will also be considered.

\section{Numerical Experiments}

In this section, we will verify the performance of the proposed algorithm, and compare it with C-SVM implemented via sequential minimal optimization [26]. The data set we used is selected in the UCI data library [3], including the IJCNN data set, SPECT heart data set and so on. We also compare the performances of different kernels, including the Gaussian kernel, $\mathrm{K}\left(\mathbf{x}_{i}, \mathbf{x}_{j}\right)=\exp \left(-\left\|\mathbf{x}_{i}-\mathbf{x}_{j}\right\|_{2}^{2} / \sigma^{2}\right)$, and the linear kernel, $\mathrm{K}\left(\mathbf{x}_{i}, \mathbf{x}_{j}\right)=\mathbf{x}_{i}^{T} \mathbf{x}_{j}$, and discuss the influence of different starting points. All the experiments are run on the Windows 7 platform of Matlab R2013a in Core(TM) i7-3770, 3.4GHz, 16GB RAM. In order to overcome the limited memory issue, we utilize the kernel caching method in the bioinfo toolbox of Matlab for the data sets which contain more than 20000 sample.

\subsection{Starting solutions}

As is discussed in Section 3.3. due to the non-convexity of ramp-LPSVM, different starting points will result in different classifiers. Considering the sparsity of the final solution, which benefits from the $l_{1}$ regularization, we will compare the following strategies for starting points:

A. Randomly set $50 \%$ of the components of the initial solution to be 0 .

B. Start from a solution of C-SVM.

Each data set is tested 10 times, and the results about the average test accuracy and its standard deviation, the average number of support vectors and training time are recorded in Table 1 and Table 2, which concern the linear kernel and the Gaussian kernel, respectively. Considering the runtime of problems with more than 10000 samples is quite long, we configure the parameters based on a smaller subset. For the other situations, we tuned the parameters by 10 -fold cross-validation.

From the results in Table 1 and 2 , we can see that in most of the cases, Strategy $\mathbf{B}$ overwhelms A in sparsity, though it requires more runtime. Generally speaking, Algorithm 2 with Gaussian kernel performs better than linear kernel in the test accuracy, while it needs more training time especially for bigger data. This is because that for small problems there is no obvious difference for Algorithm 2 to deal with different kernels, but much time will be spent on computing the kernel when the caching method is used.

\subsection{Comparison of two algorithms}

In order to verify the robustness of ramp-LPSVM solved by Algorithm 2 we randomly flip the signs of the labels of $r$ percent of the training data, where $r=0,5,10 \%$. Crossvalidation is applied in this experiment, and each experiment is repeated 10 times with starting points generated by C-SVM. The average test accuracy, number of support vectors, and training time are recorded in Table 3 and Table 4 
Table 1 Comparison of different strategies for starting points in average test accuracy (Acc.), number of support vectors (SV.) and training time (Time.) with the linear kernel.

\begin{tabular}{c|c|c|ccc}
\hline \multirow{2}{*}{ Data } & \multirow{2}{*}{$\mathrm{m}$} & \multirow{2}{*}{ Strategy } & \multicolumn{3}{|c}{ Alg.2 } \\
& & & Acc. (\%) & SV. & Time.(s) \\
\hline \multirow{2}{*}{ Spect } & \multirow{2}{*}{100} & $\mathrm{~A}$ & $91.98 \pm 0.0008$ & $\# 60$ & 0.016 \\
& & $\mathrm{~B}$ & $91.98 \pm 0.0000$ & $\# 26$ & 0.026 \\
\hline \multirow{2}{*}{ Brst. } & \multirow{2}{*}{100} & $\mathrm{~A}$ & $96.23 \pm 0.0107$ & $\# 79$ & 0.027 \\
& & $\mathrm{~B}$ & $95.20 \pm 0.8941$ & $\# 15$ & 0.018 \\
\hline \multirow{2}{*}{ Hbrn. } & \multirow{2}{*}{200} & $\mathrm{~A}$ & $68.87 \pm 0.0010$ & $\# 83$ & 0.030 \\
& & $\mathrm{~B}$ & $70.75 \pm 0.0000$ & $\# 106$ & 0.035 \\
\hline \multirow{2}{*}{ IJCNN. } & \multirow{2}{*}{1000} & $\mathrm{~A}$ & $80.86 \pm 0.0000$ & $\# 547$ & 0.247 \\
& & $\mathrm{~B}$ & $80.86 \pm 0.0004$ & $\# 215$ & 1.126 \\
\hline \multirow{2}{*}{ IJCNN. } & \multirow{2}{*}{4959} & $\mathrm{~A}$ & $82.18 \pm 0.0005$ & $\# 2041$ & 3.196 \\
& & $\mathrm{~B}$ & $86.56 \pm 2.1802$ & $\# 913$ & 4.621 \\
\hline \multirow{2}{*}{ IJCNN. } & \multirow{2}{*}{9989} & $\mathrm{~A}$ & $89.07 \pm 0.9587$ & $\# 1699$ & 15.65 \\
& & $\mathrm{~B}$ & $87.72 \pm 2.9350$ & $\# 1677$ & 17.07 \\
\hline \multirow{2}{*}{ IJCNN. } & \multirow{2}{*}{19992} & $\mathrm{~A}$ & $90.24 \pm 0.0719$ & $\# 5010$ & 48.95 \\
& & $\mathrm{~B}$ & $90.81 \pm 0.9434$ & $\# 3415$ & 47.18 \\
\hline \multirow{2}{*}{ IJCNN. } & \multirow{2}{*}{49999} & $\mathrm{~A}$ & $90.92 \pm 1.1502$ & $\# 8395$ & 365.2 \\
& & $\mathrm{~B}$ & $91.17 \pm 0.5099$ & $\# 8030$ & 373.1 \\
\hline \hline
\end{tabular}

Table 2 Comparison of different strategies for starting points in average test accuracy (Acc.), number of support vectors (SV.) and training time (Time.) with the Gaussian kernel.

\begin{tabular}{c|c|c|ccc}
\hline \multirow{2}{*}{ Data } & \multirow{2}{*}{$\mathrm{m}$} & \multirow{2}{*}{ Strategy } & \multicolumn{3}{|c}{ Alg.2 } \\
& & & Acc. (\%) & SV. & Time.(s) \\
\hline \multirow{2}{*}{ Spect } & \multirow{2}{*}{100} & $\mathrm{~A}$ & $91.98 \pm 0.0000$ & $\# 44$ & 0.016 \\
& & $\mathrm{~B}$ & $91.98 \pm 0.0000$ & $\# 28$ & 0.021 \\
\hline \multirow{2}{*}{ Brst. } & \multirow{2}{*}{100} & $\mathrm{~A}$ & $96.67 \pm 0.8879$ & $\# 63$ & 0.016 \\
& & $\mathrm{~B}$ & $94.80 \pm 0.0839$ & $\# 21$ & 0.022 \\
\hline \multirow{2}{*}{ Hbrn. } & \multirow{2}{*}{200} & $\mathrm{~A}$ & $71.70 \pm 0.0000$ & $\# 119$ & 0.035 \\
& & $\mathrm{~B}$ & $75.47 \pm 0.0000$ & $\# 111$ & 0.023 \\
\hline \multirow{2}{*}{ IJCNN. } & \multirow{2}{*}{1000} & $\mathrm{~A}$ & $80.86 \pm 0.0000$ & $\# 728$ & 0.245 \\
& & $\mathrm{~B}$ & $80.87 \pm 0.0001$ & $\# 556$ & 0.264 \\
\hline \multirow{2}{*}{ IJCNN. } & \multirow{2}{*}{4959} & $\mathrm{~A}$ & $83.15 \pm 0.0005$ & $\# 2091$ & 3.405 \\
& & $\mathrm{~B}$ & $87.34 \pm 2.0605$ & $\# 909$ & 6.089 \\
\hline \multirow{2}{*}{ IJCNN. } & \multirow{2}{*}{9989} & $\mathrm{~A}$ & $88.44 \pm 0.0439$ & $\# 6994$ & 13.06 \\
& & $\mathrm{~B}$ & $88.33 \pm 1.5710$ & $\# 1697$ & 14.94 \\
\hline \multirow{2}{*}{ IJCNN. } & \multirow{2}{*}{19992} & $\mathrm{~A}$ & $90.20 \pm 0.0957$ & $\# 9918$ & 62.50 \\
& & $\mathrm{~B}$ & $91.81 \pm 3.1322$ & $\# 3259$ & 67.18 \\
\hline \multirow{2}{*}{ IJCNN. } & \multirow{2}{*}{49999} & $\mathrm{~A}$ & $92.88 \pm 1.3625$ & $\# 9010$ & 488.2 \\
& & $\mathrm{~B}$ & $93.10 \pm 0.0008$ & $\# 8122$ & 425.3 \\
\hline \hline
\end{tabular}

From Table 3 and Table 4 we can see that Algorithm 2 trains the data faster than CSVM when the problem scale is quite small (e.g. below 1000), while for larger problems, $\mathrm{C}-\mathrm{SVM}$ is faster. Even though, there are some other advantages of Algorithm 2 over CSVM. First, the test accuracy of Algorithm 2 is better than C-SVM in most cases, since the minimization of subproblems implies global search ability to some extent. Second, within a similar test accuracy level, Algorithm 2 enjoys more sparsity which will contribute to the classification efficiency. In addition, when the number of outliers increases, their influences are less than that in C-SVM, which owes to the robustness induced by ramp loss. Besides, 
Table 3 Comparisons of C-SVM and the proposed coordinate descent algorithm with linear kernel in average test accuracy (Acc.), number of support vectors (SV.) and training time (Time.).

\begin{tabular}{|c|c|c|c|c|c|c|c|c|}
\hline \multirow{2}{*}{ Data } & \multirow[b]{2}{*}{$\mathrm{m}$} & \multirow[b]{2}{*}{$\mathrm{r}$} & \multicolumn{3}{|c|}{ C-SVM } & \multicolumn{3}{|c|}{ Alg.2. } \\
\hline & & & Acc. $(\%)$ & SV. & Time.(s) & Acc. $(\%)$ & SV. & Time.(s) \\
\hline \multirow{3}{*}{ Spect } & \multirow{3}{*}{100} & 0.00 & $91.98 \pm 0.0000$ & \#67 & 0.031 & $91.98 \pm 0.0000$ & \#26 & 0.026 \\
\hline & & 0.05 & $91.66 \pm 1.1878$ & \#69 & 0.030 & $91.98 \pm 0.0015$ & \#27 & 0.017 \\
\hline & & 0.10 & $87.70 \pm 0.4517$ & $\# 70$ & 0.030 & $91.98 \pm 0.0008$ & \#40 & 0.010 \\
\hline \multirow{3}{*}{ Brst. } & \multirow{3}{*}{100} & 0.00 & $93.55 \pm 0.0007$ & $\# 43$ & 0.031 & $95.20 \pm 0.8941$ & $\# 15$ & 0.018 \\
\hline & & 0.05 & $91.53 \pm 0.0045$ & \#50 & 0.028 & $95.40 \pm 1.1490$ & \#11 & 0.024 \\
\hline & & 0.10 & $90.69 \pm 0.0098$ & \#49 & 0.031 & $95.17 \pm 0.4983$ & \#12 & 0.022 \\
\hline \multirow{3}{*}{ Hbrn. } & \multirow{3}{*}{200} & 0.00 & $71.96 \pm 2.0138$ & $\# 110$ & 0.085 & $70.75 \pm 0.0000$ & \#106 & 0.035 \\
\hline & & 0.05 & $73.65 \pm 1.2311$ & \#118 & 0.058 & $72.53 \pm 1.7291$ & \#108 & 0.058 \\
\hline & & 0.10 & $70.10 \pm 1.3201$ & \#126 & 0.060 & $69.22 \pm 2.1584$ & \#123 & 0.058 \\
\hline \multirow{3}{*}{ IJCNN. } & \multirow{3}{*}{1000} & 0.00 & $81.68 \pm 0.0008$ & \#312 & 1.186 & $80.86 \pm 0.0004$ & $\# 215$ & 1.126 \\
\hline & & 0.05 & $78.09 \pm 0.0008$ & \#321 & 1.096 & $80.40 \pm 0.0001$ & \#244 & 1.190 \\
\hline & & 0.10 & $77.54 \pm 0.0002$ & \#299 & 1.364 & $79.94 \pm 0.0002$ & \#282 & 1.200 \\
\hline \multirow{3}{*}{ IJCNN. } & \multirow{3}{*}{4959} & 0.00 & $83.65 \pm 1.5566$ & $\# 864$ & 10.03 & $86.56 \pm 2.1802$ & \#913 & 4.621 \\
\hline & & 0.05 & $80.18 \pm 1.8731$ & \#1032 & 11.25 & $86.52 \pm 1.3450$ & \#892 & 4.563 \\
\hline & & 0.10 & $79.36 \pm 1.6542$ & \#1158 & 10.21 & $84.82 \pm 2.6946$ & \#901 & 4.349 \\
\hline \multirow{3}{*}{ IJCNN. } & \multirow{3}{*}{9989} & 0.00 & $86.44 \pm 0.0021$ & \#2134 & 15.39 & $87.72 \pm 2.9350$ & \#1677 & 17.07 \\
\hline & & 0.05 & $81.41 \pm 0.0006$ & \#2208 & 16.81 & $86.38 \pm 1.5506$ & \#1697 & 15.53 \\
\hline & & 0.10 & $80.55 \pm 0.0008$ & \#2196 & 15.73 & $85.75 \pm 1.0124$ & $\# 1740$ & 17.73 \\
\hline \multirow{3}{*}{ IJCNN. } & \multirow{3}{*}{19992} & 0.00 & $89.69 \pm 2.1189$ & \#4634 & 50.38 & $90.81 \pm 0.9434$ & \#3415 & 47.17 \\
\hline & & 0.05 & $87.21 \pm 1.0080$ & \#5102 & 51.02 & $87.50 \pm 1.4738$ & \#3306 & 48.55 \\
\hline & & 0.10 & $83.05 \pm 1.0000$ & \#5023 & 51.02 & $84.22 \pm 0.3614$ & \#3277 & 45.40 \\
\hline \multirow{3}{*}{ IJCNN. } & \multirow{3}{*}{49999} & 0.00 & $90.61 \pm 1.0212$ & \#8116 & 362.1 & $91.17 \pm 0.5099$ & \#8030 & 373.1 \\
\hline & & 0.05 & $86.15 \pm 1.9658$ & \#7903 & 361.2 & $91.08 \pm 0.6124$ & \#8120 & 411.6 \\
\hline & & 0.10 & $85.33 \pm 1.6824$ & \#8852 & 360.8 & $89.90 \pm 0.8302$ & \#8105 & 399.6 \\
\hline
\end{tabular}

Table 4 Comparisons of C-SVM and the proposed coordinate descent algorithm with Gaussian kernel in average test accuracy (Acc.), number of support vectors (SV.) and training time (Time.).

\begin{tabular}{|c|c|c|c|c|c|c|c|c|}
\hline \multirow{2}{*}{ Data } & \multirow{2}{*}{$\mathrm{m}$} & \multirow{2}{*}{$\mathrm{r}$} & \multicolumn{3}{|c|}{ C-SVM } & \multicolumn{3}{|c|}{ Alg.2] } \\
\hline & & & Acc. $(\%)$ & SV. & Time.(s) & Acc. $(\%)$ & SV. & Time.(s) \\
\hline \multirow{3}{*}{ Spect } & \multirow{3}{*}{100} & 0.00 & $91.98 \pm 2.1034$ & \#67 & 0.030 & $91.98 \pm 0.0000$ & \#28 & 0.021 \\
\hline & & 0.05 & $90.69 \pm 0.9823$ & \#70 & 0.031 & $88.88 \pm 0.5942$ & \#28 & 0.014 \\
\hline & & 0.10 & $88.30 \pm 1.2350$ & \#71 & 0.031 & $89.41 \pm 0.4957$ & \#32 & 0.015 \\
\hline \multirow{3}{*}{ Brst. } & \multirow{3}{*}{100} & 0.00 & $95.37 \pm 0.0408$ & $\# 47$ & 0.036 & $94.80 \pm 0.0839$ & $\# 21$ & 0.022 \\
\hline & & 0.05 & $93.15 \pm 0.3355$ & \#47 & 0.038 & $94.23 \pm 1.0591$ & \#21 & 0.021 \\
\hline & & 0.10 & $90.08 \pm 0.0465$ & $\# 50$ & 0.038 & $91.12 \pm 0.7639$ & $\# 18$ & 0.016 \\
\hline \multirow{3}{*}{ Hbrn.. } & \multirow{3}{*}{200} & 0.00 & $73.96 \pm 1.4381$ & $\# 133$ & 0.063 & $75.47 \pm 0.0000$ & \#111 & 0.023 \\
\hline & & 0.05 & $72.64 \pm 1.4057$ & \#118 & 0.049 & $76.57 \pm 1.9193$ & \#101 & 0.032 \\
\hline & & 0.10 & $72.64 \pm 1.7203$ & \#109 & 0.050 & $73.45 \pm 1.7928$ & \#121 & 0.032 \\
\hline \multirow{3}{*}{ IJCNN. } & \multirow{3}{*}{1000} & 0.00 & $81.89 \pm 4.0633$ & \#207 & 0.149 & $80.87 \pm 0.0001$ & $\# 556$ & 0.264 \\
\hline & & 0.05 & $77.03 \pm 3.2900$ & \#370 & 0.211 & $78.55 \pm 0.6342$ & \#219 & 0.249 \\
\hline & & 0.10 & $73.92 \pm 3.0216$ & \#420 & 0.214 & $77.02 \pm 2.3518$ & \#210 & 0.273 \\
\hline \multirow{3}{*}{ IJCNN. } & \multirow{3}{*}{4959} & 0.00 & $85.53 \pm 2.5086$ & $\# 886$ & 1.758 & $87.34 \pm 2.0605$ & $\# 909$ & 6.089 \\
\hline & & 0.05 & $84.73 \pm 2.6980$ & \#1336 & 2.746 & $86.19 \pm 2.5972$ & \#888 & 4.155 \\
\hline & & 0.10 & $84.60 \pm 2.8593$ & $\# 1008$ & 2.036 & $85.43 \pm 2.6340$ & \#890 & 3.826 \\
\hline \multirow{3}{*}{ IJCNN. } & \multirow{3}{*}{9989} & 0.00 & $86.08 \pm 3.9862$ & $\# 2302$ & 11.52 & $88.33 \pm 1.5710$ & $\# 1697$ & 14.94 \\
\hline & & 0.05 & $86.31 \pm 3.1208$ & \#2418 & 12.03 & $86.56 \pm 2.4908$ & \#1696 & 18.03 \\
\hline & & 0.10 & $71.59 \pm 3.1520$ & \#2409 & 11.98 & $86.01 \pm 1.4071$ & \#1702 & 12.98 \\
\hline \multirow{3}{*}{ IJCNN. } & \multirow{3}{*}{19992} & 0.00 & $89.23 \pm 1.8012$ & \#5986 & 40.39 & $91.96 \pm 3.1322$ & \#3259 & 67.18 \\
\hline & & 0.05 & $83.15 \pm 1.3985$ & \#5921 & 40.01 & $86.02 \pm 1.4738$ & \#3306 & 68.42 \\
\hline & & 0.10 & $81.08 \pm 1.1099$ & \#6033 & 41.52 & $84.22 \pm 0.3614$ & \#3277 & 65.91 \\
\hline \multirow{3}{*}{ IJCNN. } & \multirow{3}{*}{49999} & 0.00 & $91.12 \pm 0.0382$ & $\# 7964$ & 370.5 & $93.10 \pm 0.0008$ & $\# 8122$ & 425.3 \\
\hline & & 0.05 & $86.31 \pm 0.3421$ & \#8016 & 365.0 & $89.26 \pm 1.2645$ & \#8193 & 422.7 \\
\hline & & 0.10 & $87.08 \pm 0.6890$ & \#8125 & 371.2 & $88.24 \pm 1.0027$ & \#8108 & 422.2 \\
\hline
\end{tabular}


as the randomness exists in the proposed algorithm and also the experiments, we record the standard variance of test accuracy, which shows the stability of Algorithm 2

\section{Conclusion}

In this paper, we proposed a modified coordinate descent algorithm for ramp-LPSVM. Since the coordinate descent algorithm updates one single entry of the variable each time, the reduced one-variable piecewise linear subproblems can be solved efficiently by linearizing the concave part. In order to make it converge fast, we considered some implementation tricks to improve the efficiency of the algorithm applied. Experimental performances in robustness, sparsity and fast convergence are confirmed on benchmark data sets.

Acknowledgements This work is supported in part by the National Natural Science Foundation of China, 61134012, 61473165, and the National Basic Research Program of China, 2012CB720505. Xiaolin Huang and Johan Suykens acknowledge support from KU Leuven, the Flemish government, FWO, the Belgian federal science policy office and the European Research Council (CoE EF/05/006, GOA MANET, IUAP DYSCO, FWO G.0377.12, BIL with Tsinghua University, ERC AdG A-DATADRIVE-B)

\section{References}

1. An, L.T.H., Tao, P.D.: The DC (difference of convex functions) programming and DCA revisited with DC models of real world nonconvex optimization problems. Annals of Operations Research 133(1-4), 23-46 (2005)

2. Aronszajn, N.: Theory of reproducing kernels. Transactions of the American Mathematical Society 68(3), 337-404 (1950)

3. Bache, K., Lichman, M.: UCI machine learning repository (2013). URL http://archive.ics.uci.edu/ml

4. Bartlett, P.L., Mendelson, S.: Rademacher and Gaussian complexities: Risk bounds and structural results. Journal of Machine Learning Research 3, 463-482 (2003)

5. Boser, B.E., Guyon, I.M., Vapnik, V.N.: A training algorithm for optimal margin classifiers. In: Proceedings of the Fifth Annual Workshop on Computational Learning Theory, COLT '92, pp. 144-152. ACM, New York, NY, USA (1992)

6. Brooks, J.P.: Support vector machines with the ramp loss and the hard margin loss. Operations Research 59(2), 467-479 (2011)

7. Carrizosa, E., Nogales-Gmez, A., Romero Morales, D.: Heuristic approaches for support vector machines with the ramp loss. Optimization Letters 8(3), 1125-1135 (2014)

8. Chang, K.W., Hsieh, C.J., Lin, C.J.: Coordinate descent method for large-scale L2-loss linear support vector machines. Journal of Machine Learning Research 9, 1369-1398 (2008)

9. Collobert, R., Sinz, F., Weston, J., Bottou, L.: Trading convexity for scalability. In: Proceedings of the 23rd international conference on Machine learning, pp. 201-208. ACM (2006)

10. Cortes, C., Vapnik, V.: Support-vector networks. Machine Learning 20(3), 273-297 (1995)

11. Friedman, J., Hastie, T., Tibshirani, R.: Regularization paths for generalized linear models via coordinate descent. Journal of Statistical Software 33(1), 1 (2010)

12. Fung, G.M., Mangasarian, O.L.: A feature selection newton method for support vector machine classification. Computational Optimization and Applications 28(2), 185-202 (2004)

13. Hildreth, C.: A quadratic programming procedure. Naval Research Logistics Quarterly 4(1), 79-85 (1957)

14. Hsieh, C.J., Chang, K.W., Lin, C.J., Keerthi, S.S., Sundararajan, S.: A dual coordinate descent method for large-scale linear SVM. In: Proceedings of the 25th International Conference on Machine Learning, ICML '08, pp. 408-415. ACM, New York, NY, USA (2008)

15. Huang, X., Shi, L., Suykens, J.A.K.: Ramp loss linear programming support vector machine. Journal of Machine Learning Research 15, 2185-2211 (2014)

16. Huang, X., Xu, J., Mu, X., Wang, S.: The hill detouring method for minimizing hinging hyperplanes functions. Computers \& Operations Research 39(7), 1763-1770 (2012) 
17. Lee, C.P., Lin, C.J.: A study on L2-loss (squared hinge-loss) multiclass SVM. Neural Computation 25(5), 1302-1323 (2013)

18. Liu, Y., Helen Zhang, H., Park, C., Ahn, J.: Support vector machines with adaptive $L_{q}$ penalty. Computational Statistics \& Data Analysis 51(12), 6380-6394 (2007)

19. Liu, Y., Wu, Y.: Optimizing $\psi$-learning via mixed integer programming. Statistica Sinica 16(2), 441 (2006)

20. Luo, Z., Tseng, P.: On the convergence of the coordinate descent method for convex differentiable minimization. Journal of Optimization Theory and Applications 72(1), 7-35 (1992)

21. Mangasarian, O.L.: A finite Newton method for classification. Optimization Methods and Software 17(5), 913-929 (2002)

22. Mangasarian, O.L.: Exact 1-norm support vector machines via unconstrained convex differentiable minimization. Journal of Machine Learning Research 7, 1517-1530 (2006)

23. Mangasarian, O.L., Musicant, D.R.: Successive overrelaxation for support vector machines. IEEE Transactions on Neural Networks 10(5), 1032-1037 (1999)

24. Mazumder, R., Friedman, J.H., Hastie, T.: Sparsenet: Coordinate descent with nonconvex penalties. Journal of the American Statistical Association 106(495), 1125-1138 (2011)

25. McAllester, D., Keshet, J.: Generalization bounds and consistency for latent structural probit and ramp loss. In: Advances in Neural Information Processing Systems 24, pp. 2205-2212 (2011)

26. Platt, J.: Fast training of support vector machines using sequential minimal optimization. In: B. Schölkopf, C.J. Burges, A.J. Smola (eds.) Advances in Kernel Methods: Support Vector Learning, pp. 185-208. MIT press (1999)

27. Schmidt, M., Fung, G., Rosales, R.: Fast optimization methods for L1 regularization: A comparative study and two new approaches. In: Machine Learning: European Conference on Machine Learning 2007, vol. 4701, pp. 286-297. Springer Berlin Heidelberg (2007)

28. Shalev-Shwartz, S., Tewari, A.: Stochastic methods for $l_{1}$-regularized loss minimization. Journal of Machine Learning Research 12, 1865-1892 (2011)

29. Shao, Y.H., Deng, N.Y.: A coordinate descent margin based-twin support vector machine for classification. Neural Networks 25(0), 114 - 121 (2012)

30. Suykens, J.A.K., Vandewalle, J.: Least squares support vector machine classifiers. Neural Processing Letters 9(3), 293-300 (1999)

31. Tseng, P., Yun, S.: A coordinate gradient descent method for nonsmooth separable minimization. Mathematical Programming 117(1-2), 387-423 (2009)

32. Wang, L., Jia, H., Li, J.: Training robust support vector machine with smooth ramp loss in the primal space. Neurocomputing 71(13), 3020-3025 (2008)

33. Wu, Y., Liu, Y.: Robust truncated hinge loss support vector machines. Journal of the American Statistical Association 102(479), 974-983 (2007)

34. Xu, L., Crammer, K., Schuurmans, D.: Robust support vector machine training via convex outlier ablation. In: Proceedings of the 21st National Conference on Artificial Intelligence, AAAI'06, pp. 536-542. AAAI Press (2006)

35. Yuan, G.X., Chang, K.W., Hsieh, C.J., Lin, C.J.: A comparison of optimization methods and software for large-scale L1-regularized linear classification. Journal of Machine Learning Research 11, 3183-3234 (2010)

36. Zhu, J., Rosset, S., Tibshirani, R., Hastie, T.J.: 1-norm support vector machines. In: Advances in Neural Information Processing Systems 16, pp. 49-56. MIT Press (2004) 